\title{
Maintenance Therapy with Tegafur-gimeracil-oteracil After First-line Chemotherapy in Stage IV Gastric Cancer: A Retrospective Study
}

\author{
Kena Zhou ${ }^{1,2, \dagger}$, Anjing Zhao ${ }^{1, \dagger}$, Bin Wang ${ }^{1}$, Jie Li ${ }^{1}$, Yingyi Zhang ${ }^{1}$, Meihong Wu ${ }^{1, ~ *, ~}$ \\ Xianbao Zhan,"* \\ ${ }^{1}$ Department of Oncology, Changhai Hospital Affiliated to Naval Military Medical University, Shanghai, China \\ ${ }^{2}$ Department of Gastroenterology, Ningbo No. 9 Hospital, Ningbo, China
}

Email address:

doctorwmh@163.com (Meihong Wu), zhanxianbao@csco.org.cn (Xianbao Zhan)

${ }^{*}$ Corresponding author

$\dagger$ Kena Zhou and Anjing Zhao are co-first authors.

\section{To cite this article:}

Kena Zhou, Anjing Zhao, Bin Wang, Jie Li, Yingyi Zhang, Meihong Wu, Xianbao Zhan. Maintenance Therapy with Tegafur-gimeracil-oteracil After First-line Chemotherapy in Stage IV Gastric Cancer: A Retrospective Study. American Journal of Clinical and Experimental Medicine. Vol. 9, No. 5, 2021, pp. 168-173. doi: 10.11648/j.ajcem.20210905.17

Received: September 22, 2021; Accepted: October 15, 2021; Published: October 19, 2021

\begin{abstract}
Introduction: The feasibility, efficacy and safety of maintenance chemotherapy (MCT) after first-line therapy in advanced gastric cancer (AGC) remain obscure. This study aims to explore the efficacy and safety of tegafur-gimeracil-oteracil MCT in stage IV GC. Methods: Seventeen patients with stage IV GC had been involved in this study from the Department of Oncology of Changhai Hospital from January $1^{\text {st }}, 2017$ to August $31^{\text {st }}$, 2021. After 6-8 cycles of first-line chemotherapy with SOX (tegafur-gimeracil-oteracil combined with oxaliplatin) or DS (docetaxel combined with tegafur-gimeracil-oteracil) MCT was administered with tegafur-gimeracil-oteracil which continued until disease progression or intolerable adverse effects or death. The PFS, OS, DCR and adverse effects were analyzed. Results: Among 17 patients with stage IV GC, 11 patients received prior SOX-based first-line therapy, 6 patients with TS regimen. The best curative effect of first-line therapy were CR in 1 case, PR in 2 and SD in 14. DCR was 87.5\% after tegafur-gimeracil-oteracil MCT, and the median PFS was 13.5 months, the median OS was 23 months. The main adverse effects of tegafur-gimeracil-oteracil MCT were grade 1-3 hematologic and grade 1 fatigue, gastrointestinal symptoms. There were no treatment-related deaths or toxic effects of grade 4 .
\end{abstract}

Keywords: Gastric Cancer, Tegafur-gimeracil-oteracil, Maintenance Chemotherapy

\section{Introduction}

Gastric cancer (GC) is one of the most common malignant tumors in the world, which is more severe in China. In 2020, there was 480,000 new cases of GC in China, leading to a mortality of 370,000 , which accounted for $48 \%$ worldwide [1] As an extremely malignant tumor, the prognosis of GC was directly associated with tumor stage [2]. The 5-year survival rates of stage I-IV GC were about $86.7 \%, 73.2 \%, 44.3 \%$, and $13.4 \%$, respectively $[3,4]$. Due to a lack of early symptoms and low screening rate in general, the proportion of AGC in China remains in a high level. According to the epidemiological data of the Chinese Gastrointestinal
Oncology Surgery Union [5], it was suggested that stage IV GC contributed $9.7 \%$ in Chinese GC patients. And the median PFS and OS were 4 months and 11 months [6].

Palliative chemotherapy, targeted and immune therapy were currently the mainstays for stage IV GC referring to the recommendations of $\mathrm{NCCN}$ and $\mathrm{CSCO} \mathrm{GC}$ guidelines. However, the effect of first-line regimen was still not satisfactory, the median PFS, OS and 5-year survival rate were only 7.5 months, 13 months and 24\% [7, 8]. Moreover, on account of rapid development of drug resistance, disease progression often occurs after a median of 4-6 cycles of chemotherapy [9]. The response of first-line therapy is significantly associated with overall prognosis in AGC. Therefore, how to optimize the effect of first-line therapy is 
catching more and more attention.

MCT after first-line treatment is well established in other tumor types such as lung cancer, breast cancer and colorectal cancer [10-14]. Still there is no consensus or evidence-based medicine of MCT in AGC. Exploring the regimen, efficacy and safety of MCT in AGC is helpful to optimize the effect of first-line chemotherapy, even more to modify the standard strategy in AGC treatment. For our previous clinical practices, we have tried to apply tegafur-gimeracil-oteracil MCT after first-line induction for stage IV GC patients. Here we reported our findings as follows.

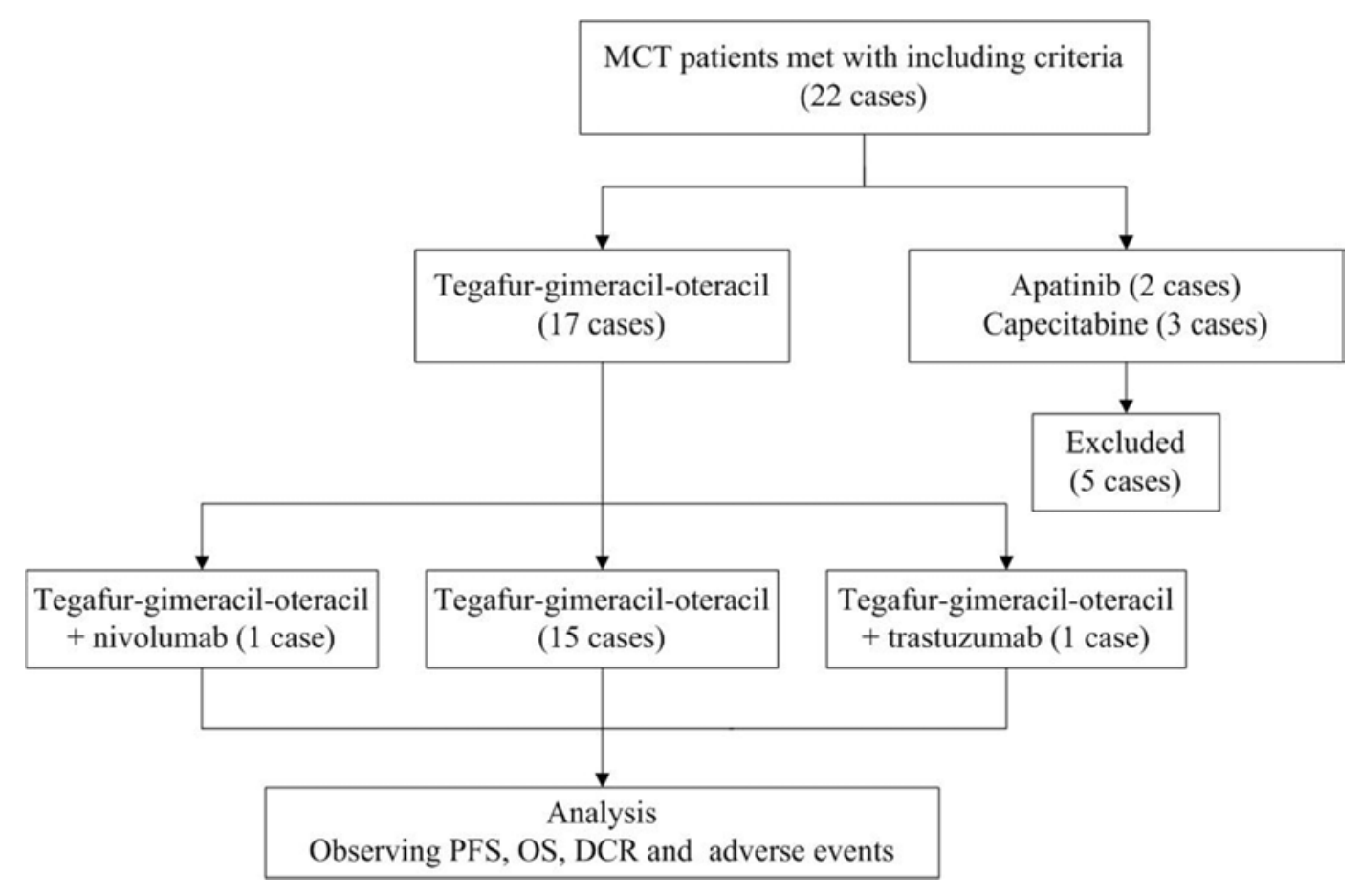

Figure 1. Flow chart of 17 included cases with tegafur-gimeracil-oteracil.

\section{Materials and Methods}

\subsection{Patient Eligibility}

The study included 22 patients with stage IV GC who had been receiving MCT in Shanghai Changhai Hospital from January $1^{\text {st }}, 2017$ to August $31^{\text {st }}, 2021$. All patients were confirmed to be stage IV GC by pathological and imaging examinations. Among them, 17 patients after disease control $(\mathrm{CR}+\mathrm{PR}+\mathrm{SD})$ were included in this study, who received tegafur-gimeracil-oteracil MCT after the first-line standard chemotherapy. The procedure for selecting subjects for enrollment were displayed in the following flow chart (shown in Figure 1). All cases included in this study meet the following conditions: pathologically diagnosed stage IV GC; being measurable or evaluable lesions; expected survival time $\geq 12$ weeks; Eastern cooperative oncology group performance status (ECOG PS) score $<2$; no other significant impact on survival apart from AGC.

\subsection{First-line Therapy}

\subsubsection{Standard First-line Chemotherapy Regimens}

SOX: Oxaliplatin $130 \mathrm{mg} / \mathrm{m}^{2}$ iv $\mathrm{d} 1$, tegafur-gimeracil-oteracil $40 \mathrm{mg} / \mathrm{m}^{2}$ po bid d1-14. Each cycle is repeated on day 22 .
TS: Docetaxel $75 \mathrm{mg} / \mathrm{m}^{2}$ iv d1, tegafur-gimeracil-oteracil $40 \mathrm{mg} / \mathrm{m}^{2}$ po bid d1-14. Each cycle is repeated on day 22 .

In addition, trastuzumab is combined in patients with Human epidermal growth factor receptor-2 (HER2) positive GC. The initial loading dose is $8 \mathrm{mg} / \mathrm{kg}$, followed by $6 \mathrm{mg} / \mathrm{kg}$. Each cycle is repeated on day 22 . The first infusion time is about 90 minutes. If the patient is well tolerated during the first time, the subsequent infusion can be 30-60 minutes. If there is a delay or interruption in the treatment process, the maintenance dose can be used directly if the delay time is less than 1 week, and the loading dose should be re-introduced if the delay period is more than 1 week.

Nivolumab is combined if HER2 overexpression negative with PD L1 CPS $\geq 5$ according to NCCN Guidelines Version 4.2021 for GC. The dosage is applied as $3 \mathrm{mg} / \mathrm{kg}$ iv $60 \mathrm{~min}$. Each cycle is repeated on day 15 .

\subsubsection{MCT Regimens}

Tegafur-gimeracil-oteracil $40 \mathrm{mg} / \mathrm{m}^{2}$ po bid d1-14. Each cycle is repeated on day 22. One patient with HER2 positive combined with trastuzumab. And 1 case combined with nivolumab considering NCCN GC guidelines and the patient's strong willingness.

\subsection{Evaluation Criteria and Follow-up}

The 17 patients in the study were comprehensively assessed 
to achieve disease control after 6-8 cycles of standard first-line chemotherapy, and then tegafur-gimeracil-oteracil MCT was administered. In order to evaluate the efficacy and safety during MCT, regular blood tests were tested to reflect hematological adverse effects, CT or MRI and other imaging methods to specify the size of the lesion. The frequency of follow-up for patients was approximately every 3 months, adopting ways of inpatient, outpatient, and telephone. The deadline for follow-up was August $31^{\text {st }}, 2021$.

Response Evaluation Criteria in Solid Tumors (RECIST 1.1) was used to evaluate the efficacy. The results could be signified as the following four parts. Complete remission (CR) referred to that all target lesions disappear, and the short diameter of all pathological lymph nodes was reduced to $<10 \mathrm{~mm}$. Partial remission (PR) meant that the sum of target lesion diameters was reduced by at least $30 \%$ from the baseline level. Disease control (SD) denoted the situation between PR and PD. Disease progression (PD) represents the minimum value of the sum of the diameters of all target lesions measured during the entire treatment process increased by at least $20 \%$, or one or more new lesions should appear.

The disease control rate (DCR) was the percentage of $\mathrm{PR}+\mathrm{SD}$ patients in the total patients. PFS was the progression-free survival time. OS was the overall survival time. The assessment of the adverse effect of MCT was strictly in accordance with the National Cancer Institute Common Toxicity Criteria version 3.0, grouping into grade I to IV.

\subsection{Data Analysis}

Characteristic statistics were collected and sorted in Microsoft Excel. The flow chart was drawn using Microsoft Office Visio. Non-normally distributed data is represented by median, and countable data is represented by proportion (\%) when appropriate. PFS and OS were estimated using the Kaplan-Meier survival analysis and Log-rank test. All statistical analyses were performed in SPSS software (version 13.0), including figure drawing.

\section{Results}

\subsection{Patients' Clinicopathological Characteristics}

Table 1. Characteristics of patients included in this study $(N=17)$.

\begin{tabular}{|c|c|}
\hline Characteristics & Cases (\%) \\
\hline \multicolumn{2}{|l|}{ Age (year) } \\
\hline Median & 56 \\
\hline Range & $28-69$ \\
\hline \multicolumn{2}{|l|}{ Gender } \\
\hline Male & $8(47.1 \%)$ \\
\hline Female & $9(52.9 \%)$ \\
\hline \multicolumn{2}{|l|}{ Differentiation types } \\
\hline Poorly differentiated & $7(41.2 \%)$ \\
\hline Moderate to poorly differentiated & $2(11.8 \%)$ \\
\hline Moderate differentiation & $3(17.6 \%)$ \\
\hline Unknown & $4(23.5 \%)$ \\
\hline \multicolumn{2}{|l|}{ Sites of metastasis } \\
\hline Pelvic (including ovary and omentum) & $14(82.3 \%)$ \\
\hline Liver, lymph nodes & $2(11.8 \%)$ \\
\hline No surgery & $2(11.8 \%)$ \\
\hline Exploratory biopsy & $6(35.3 \%)$ \\
\hline Palliative radical surgery & $9(52.9 \%)$ \\
\hline \multicolumn{2}{|l|}{ Maintenance therapy regimen } \\
\hline tegafur-gimeracil-oteracil & $15(88.2 \%)$ \\
\hline tegafur-gimeracil-oteracil + trastuzumab & $1(5.9 \%)$ \\
\hline tegafur-gimeracil-oteracil + nivolumab & $1(5.9 \%)$ \\
\hline
\end{tabular}

This study included 17 patients with stage IV GC who underwent tegafur-gimeracil-oteracil MCT in the Oncology Department of Shanghai Changhai Hospital from January $1^{\text {st }}$, 2017 to August $31^{\text {st }}, 2021$. The final follow-up time is August $31^{\text {st }}, 2021$. Baseline of patients' demographics and disease characteristics is shown in Table 1.

Among the 17 patients with stage IV GC in MCT, there were eight men and nine women. The median age is 56 years old $(\mathrm{P} 25=44, \mathrm{P} 75=63.5)$. According to the degree of pathological differentiation, one case was signet ring cell carcinoma, seven cases were poorly differentiated, two cases were moderate-poorly differentiated, three cases were moderate differentiated, and four cases were unknown. 82.3\% of the enrolled stage IV GC patients had pelvic metastasis, including ovarian and omental metastasis. Six patients underwent exploratory abdominal surgery, nine patients underwent palliative radical surgery, and two patients received conservative medical treatment throughout the course. The main MCT drug was tegafur-gimeracil-oteracil, of which one case combined with trastuzumab targeted therapy for 
HER2-positive, and also 1 case combined immunotherapy with nivolumab.

\subsection{Efficacy}

All 17 patients received tegafur-gimeracil-oteracil MCT after standard first-line treatment for stage IV GC. After follow-up, the standard first-line chemotherapy curative effect was one case with CR (5.9\%), two cases (11.8\%) with PR and fourteen cases $(82.3 \%)$ with SD. One patient who achieved
CR in first-line chemotherapy was evaluated as continued CR during tegafur-gimeracil-oteracil MCT + nivolumab MCT. And the other 15 patients with standard treatment reaching PR or SD remain SD as the best efficacy in MCT process. As for the MCT, 17 patients received 2 to 32 cycles (median 8 cycles), the median PFS was 13.5 months, and the median OS was 23 months. The survival curves of PFS and OS of 17 patients were drawn (shown in Figure 2).
OS Curve

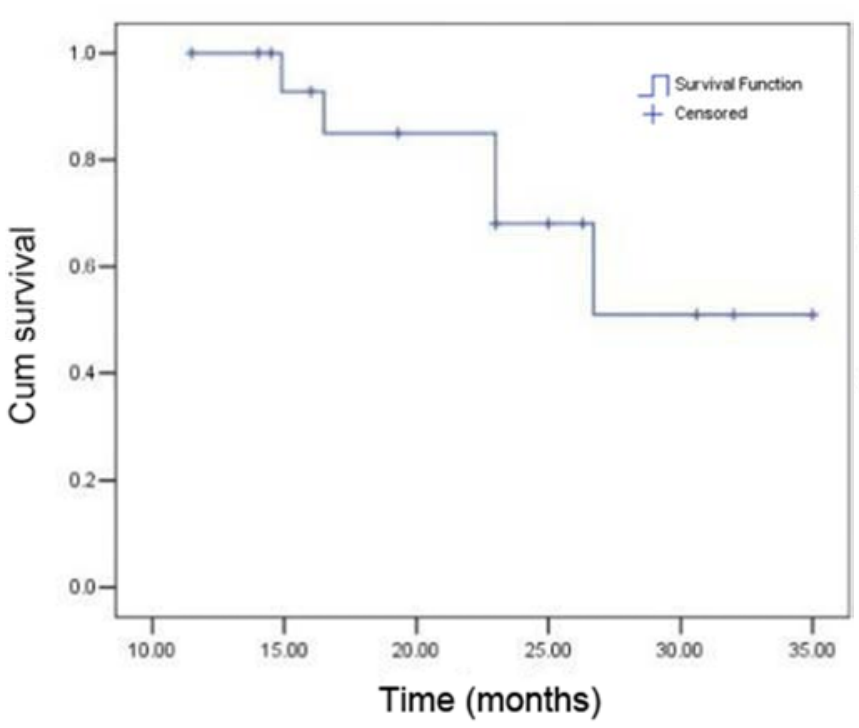

PFS curve

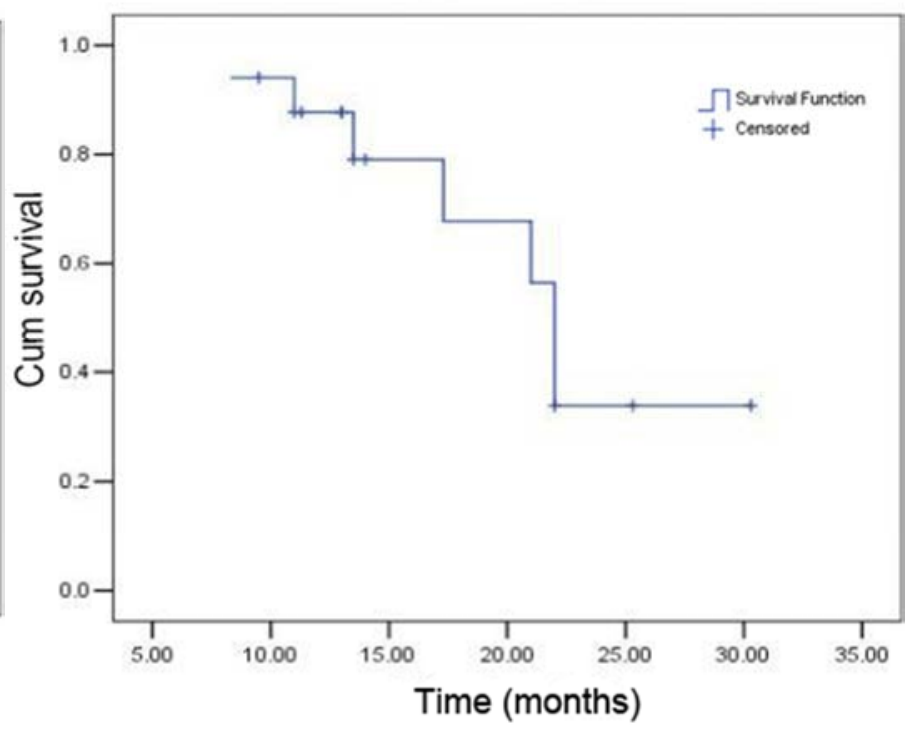

Figure 2. PFS and OS survival curves of the 17 patients.

Two patients were in PD at the first assessment of MCT and entered second-line chemotherapy. The DCR of MCT was $88.2 \%$. Totally five cases died with the median OS of 11.3 months. All died cases were poorly differentiated and accompanied with abdominal metastasis. Twelve patients survived long-term until the final follow-up. Among them, two patients discontinued taking drugs after tegafur-gimeracil-oteracil MCT for 2 years, and were verified to be in the condition of SD during regular evaluation. One case was a 55-year-old female with liver metastasis, and one case was a 67-year-old male with abdominal metastasis, both the two patients had normal blood tumor markers during the whole treatment process. Another good-ending case was a 68-year-old male with peritoneal metastasis, whose curative effect reached CR after first-line chemotherapy. So far, he has been applied with tegafur-gimeracil-oteracil combined with nivolumab as MCT for 8 months, and his condition is stable under regular examination.

\subsection{Safety}

Our study found that the most common adverse effect during MCT was hematologic, especially from grade 1 to 2 , since four patients had leukopenia and thrombocytopenia. Two patients developed fatigue during MCT and one case had gastrointestinal symptoms of mild nausea, vomiting, and diarrhea. And one patient with MCT of tegafur-gimeracil-oteracil combined with trastuzumab developed grade I bilirubin elevation and grade III leukopenia. Fortunately, these adverse effects could be cured spontaneously during the intermittent period or be relieved after supportive handling. No grade IV adverse effects and treatment-related deaths occurred during MCT. The detailed safety assessment of tegafur-gimeracil-oteracil MCT is exhibited in Table 2.

Table 2. Toxicity for 17 assessable patients with tegafur-gimeracil-oteracil.

\begin{tabular}{lllll}
\hline \multirow{2}{*}{ Toxicity } & Cases & & & \\
\cline { 2 - 4 } & Grade 1 & Grade 2 & Grade 3 & Grade 4 \\
\hline Hematologic & 2 & 2 & 1 & 0 \\
Fatigue & 2 & 0 & 0 & 0 \\
Nausea, Vomiting, Diarrhea & 1 & 0 & 0 & 0 \\
Bilirubin damage & 1 & 0 & 0 & 0 \\
\hline
\end{tabular}




\section{Discussion}

There is no definitive conclusion on the feasibility, efficacy and safety of the best regimen in MCT after the first-line standard treatment of AGC. A research conducted by Chen et al. reported that the median PFS and OS of patients with supportive treatment after first-line chemotherapy for AGC were 6.8 months and 11.7 months respectively [15]. In a randomized controlled study of 121 patients, the median PFS and OS of patients in the continuous chemotherapy group after standard first-line chemotherapy were 10.5 months and 22.6 months, while the median PFS and OS of patients in the observation group who stopped chemotherapy was 7.2 months and 22.7 months [16]. In our study, patients with stage IV GC received tegafur-gimeracil-oteracil MCT after disease control from standard first-line chemotherapy of SOX or TS regimens. The median PFS and median OS for them is 13.5 months and 23 months, which are longer than other previous studies.

Researches by predecessors have showed that tegafur-gimeracil-oteracil has adverse effects such as neutropenia, thrombocytopenia, anemia, and oral mucositis [17]. Japanese scholars have also found that tegafur-gimeracil-oteracil could cause anorexia, anemia, and fatigue in adjuvant treatment during perioperative period [18]. In our study, we observed 17 tegafur-gimeracil-oteracil MCT patients of AGC, and the main toxic effects were grade 1-3 hematologic, grade 1 bilirubin damage, mild gastrointestinal reactions and fatigue. These adverse effects could be relieved after intermittent or after supportive measures. Our results indicated that adverse effect of tegafur-gimeracil-oteracil in MCT of AGC could be well tolerated.

However, our study has some limitations. Firstly, it is a single-center retrospective study without a control group. Therefore, multi-center studies are needed to replicate and validate our findings. Secondly, the number of patients enrolled in the group is indeed small. In the near future, we plan to expand the sample size for more evidence.

\section{Conclusion}

In summary, our study preliminarily suggests that MCT is likely to be a feasible strategy for AGC. Moreover, tegafur-gimeracil-oteracil was proved to have good efficacy and safety in MCT of stage IV GC, while obtaining longer PFS and OS. These results have promising evidence on measurements taken in treating AGC. Since this study is retrospective and the scale of patients enrolled is small, it is worthy of a prospective study with a larger sample in the future. In the actual clinical time, the choice of MCT needs to consider the patient's condition, physical function, drug tolerance, expectations for the quality of life, and economic status. Individualized treatment of GC can not only prolong the survival time of patients, but also improve the quality of life of patients and bring huge benefits.

\section{Author Contributions}

Kena Zhou and Anjing Zhao carried out the study and did analysis. Bin Wang, Jie Li. Anjing Zhao, Yingyi Zhang collected the data. Kena Zhou and Meihong Wu drafted the manuscript together. Meihong $\mathrm{Wu}$ and Xianbao Zhan participated in paper design and supervised the draft. All authors have read and approved the final manuscript.

\section{Conflict of Interest Statement}

The authors declare that they have no competing interests.

\section{Data Availability Statement}

All data details are available from the corresponding authors.

\section{Acknowledgements}

This study is financially supported by grants from the Foundation for the Detection of immune checkpoint expression in circulating tumor cells of gastric cancer for early prognosis and prediction of the efficacy of immune checkpoint inhibitors/202040412.

\section{References}

[1] Siegel RL, Miller KD, Jemal A. Cancer statistics, 2020. CA: a cancer journal for clinicians. $2020 \mathrm{Jan} ; 70$ (1): 7-30.

[2] Zhang M, Ding C, Xu L, Ou B, Feng S, Wang G, et al. Comparison of a Tumor-Ratio-Metastasis Staging System and the 8th AJCC TNM Staging System for Gastric Cancer. Frontiers in oncology. 2021; 11: 595421.

[3] Ji XK, Madhurapantula SV, He G, Wang KY, Song CH, Zhang JY, et al. Genetic variant of cyclooxygenase-2 in gastric cancer: More inflammation and susceptibility. World journal of gastroenterology. $2021 \mathrm{Jul}$ 28; 27 (28): 4653-66.

[4] Suzuki S, Takahashi A, Ishikawa T, Akazawa K, Katai H, Isobe Y, et al. Surgically treated gastric cancer in Japan: 2011 annual report of the national clinical database gastric cancer registry. Gastric Cancer. 2021 May; 24 (3): 545-66.

[5] Wang Yinkui. The current status of diagnosis and treatment of early gastric cancer in my country: Enlightenment from data from China Gastrointestinal Oncology Surgery Union. Chinese Journal of Gastrointestinal Surgery. 2018; 21 (2).

[6] Pommergaard HC, Nielsen K, Sorbye H, Federspiel B, Tabaksblat EM, Vestermark LW, et al. Surgery of the primary tumour in 201 patients with high-grade gastroenteropancreatic neuroendocrine and mixed neuroendocrine-non-neuroendocrine neoplasms. Journal of neuroendocrinology. 2021 May; 33 (5): e12967.

[7] Lui A, Mulder K, Brezden-Masley C, Vickers M, Monzon J, Kennecke H, et al. A multicentre, open-label phase II study of Irinotecan, capecitabine (Xeloda $\left.{ }^{\circledR}\right)$, and Oxaliplatin (IXO) as first-line treatment in patients with metastatic gastric or gastroesophageal junction (GEJ) adenocarcinoma. Investigational new drugs. 2018 Aug; 36 (4): 674-82. 
[8] Solaini L, Ministrini S, Bencivenga M, D'Ignazio A, Marino E, Cipollari $\mathrm{C}$, et al. Conversion gastrectomy for stage IV unresectable gastric cancer: a GIRCG retrospective cohort study. Gastric Cancer. 2019 Nov; 22 (6): 1285-93.

[9] Yao Y, Deng R, Liao D, Xie H, Zuo J, Jia Y, et al. Maintenance treatment in advanced HER2-negative gastric cancer. Clinical $\&$ translational oncology: official publication of the Federation of Spanish Oncology Societies and of the National Cancer Institute of Mexico. 2020 Dec; 22 (12): 2206-12.

[10] Lal R, Dickson J, Cunningham D, Chau I, Norman AR, Ross PJ, et al. A randomized trial comparing defined-duration with continuous irinotecan until disease progression in fluoropyrimidine and thymidylate synthase inhibitor-resistant advanced colorectal cancer. Journal of clinical oncology: official journal of the American Society of Clinical Oncology. 2004 Aug 1; 22 (15): 3023-31.

[11] Tournigand C, Cervantes A, Figer A, Lledo G, Flesch M, Buyse M, et al. OPTIMOX1: a randomized study of FOLFOX4 or FOLFOX7 with oxaliplatin in a stop-and-Go fashion in advanced colorectal cancer--a GERCOR study. Journal of clinical oncology: official journal of the American Society of Clinical Oncology. 2006 Jan 20; 24 (3): 394-400.

[12] Chibaudel B, Maindrault-Goebel F, Lledo G, Mineur L, André $\mathrm{T}$, Bennamoun $\mathrm{M}$, et al. Can chemotherapy be discontinued in unresectable metastatic colorectal cancer? The GERCOR OPTIMOX2 Study. Journal of clinical oncology: official journal of the American Society of Clinical Oncology. 2009 Dec 1; 27 (34): 5727-33.

[13] Ciuleanu T, Brodowicz T, Zielinski C, Kim JH, Krzakowski M,
Laack E, et al. Maintenance pemetrexed plus best supportive care versus placebo plus best supportive care for non-small-cell lung cancer: a randomised, double-blind, phase 3 study. Lancet (London, England). 2009 Oct 24; 374 (9699): 1432-40.

[14] Huang H, Jiang Z, Wang T, Zhang S, Bian L, Cao Y, et al. Single-agent capecitabine maintenance therapy after response to capecitabine-based combination chemotherapy in patients with metastatic breast cancer. Anti-cancer drugs. 2012 Aug; 23 (7): 718-23.

[15] Chen J, Shen W, Xia J, Xu R, Zhu M, Xu M. [Effect of S-1 maintenance chemotherapy following DCF regimen in patients with advanced gastric cancer]. Nan fang yi ke da xue xue bao= Journal of Southern Medical University. 2014 Jun; 34 (7): 1057-60.

[16] Park SR, Kim MJ, Nam BH, Kim CG, Lee JY, Cho SJ, et al. A randomised phase II study of continuous versus stop-and-go S-1 plus oxaliplatin following disease stabilisation in first-line chemotherapy in patients with metastatic gastric cancer. European journal of cancer (Oxford, England: 1990). 2017 Sep; 83: $32-42$.

[17] Zhang Q, Qian Y, Yin Y. Comparison of S-1-based vs. capecitabine-based adjuvant chemotherapy for patients with gastric cancer: a systematic review and meta-analysis. European journal of clinical pharmacology. $2021 \mathrm{Jul} 17$.

[18] Serizawa A, Kuramochi H, Taniguchi K, Ota M, Katagiri S, Yamada T, et al. Phase II study of neoadjuvant chemotherapy with S-1 plus oxaliplatin for gastric cancer clinical T4 or N2-3. Medical oncology (Northwood, London, England). 2021 Jul 24; 38 (9): 98. 